\title{
Assistência de enfermagem no cuidado de feridas na atenção primária em saúde: revisão integrativa
}

\author{
Nursing care in wound care in primary health care: integrative review
}

\begin{abstract}
Atención de enfermería en atención de heridas en atención primaria de salud: revisión integradora
\end{abstract}

Márcia Beatriz Viana de Sousa ${ }^{1 *}$, Alexsandra Maria Ferreira de Araújo Bezerra ${ }^{2}$, Cleuson Vieira Costa $^{3}$, Edilene Bispo Gomes ${ }^{4}$, Hadsan Taiana Aleixo da Fonseca ${ }^{5}$, Odileia Borges Quaresma ${ }^{6}$, Odemar Raimundo Gonçalves Baena Júnior ${ }^{7}$, Silvio Douglas Medeiros Costa ${ }^{5}$, Suellen Patrícia Sales da Costa Loureiro ${ }^{8}$, Suenildo Messias da Silva ${ }^{9}$.

\section{RESUMO}

Objetivo: Verificar a importância da assistência de enfermagem no cuidado de feridas na atenção primária em saúde. Métodos: Trata-se de uma revisão integrativa da literatura, realizada nas bases de dados Public Library U. S. National Library of Medicine (PubMed) e Biblioteca Virtual em Saúde (BVS) por meio da associação de descritores, tendo como critério de inclusão estudos primários, disponíveis na integra, publicados nos últimos 5 anos, em qualquer idioma que atendessem a temática. Resultados: Realizou-se a análise de 11 artigos que discutiram a importância da assistência de enfermagem no cuidado a feridas na atenção primária em saúde, na qual observou-se que a assistência é realizada de forma empírica e sem uso de protocolos, identificou a escassez de adequadas para a realização do tratamento de feridas, bem como o baixo conhecimento dos profissionais referente ao tratamento, conduta, escolha de curativo e assistência prestada aos pacientes, associadas à falta de capacitação dos profissionais e às deficiências nos seu processo formativo. Considerações finais: Os profissionais da enfermagem desempenham um papel de extrema importância no tratamento das feridas orienta, executa e supervisiona a equipe de enfermagem na realização de curativos, atuando na prevenção, avaliação e indicação do tratamento adequado para a lesão.

Palavras-chave: Ferimentos e lesões, Cicatrização, Centros de saúde.

\section{ABSTRACT}

Objective: To verify the importance of nursing care in wound care in primary health care. Methods: This is an integrative literature review, carried out in the Public Library US National Library of Medicine (PubMed) and Virtual Health Library (VHL) databases through the association of descriptors, with primary studies as inclusion criteria, available in full, published in the last 5 years, in any language that met the theme. Results: An analysis of 11 articles was carried out that discussed the importance of nursing care in wound care in primary health care, in which it was observed that care is performed empirically and without the use of protocols, identified the scarcity adequate to perform the treatment of wounds, as well as the low

\footnotetext{
${ }^{1}$ Faculdade Integral Diferencial (FACID), Teresina - PI. *E-mail: marciabv123@hotmail.com

2Universidade Federal do Piauí (UFPI), Teresina - PI.

${ }^{3}$ Faculdade Estácio Macapá (FAMAP), Belém - PA.

${ }^{4}$ Universitário do Estado do Pará (CESUPA), Belém - PA.

5Universidade da Amazônia (UNAMA), Belém - PA.

${ }^{6}$ Escola Superior da Amazônia (ESAMAZ), Belém - PA.

${ }^{7}$ Centro Universitário Metropolitana da Amazônia (UniFAMAZ), Belém - PA.

8 Universidade Estadual do Pará (UEPA), Belém - PA.

${ }^{9}$ Faculdade Maurício de Nassau (FMN), Belém - PA.
} 
knowledge of professionals regarding treatment, conduct, choice of dressing and assistance provided to patients, associated with the lack of training of professionals and deficiencies in their training process. Final considerations: Nursing professionals play an extremely important role in the treatment of wounds guides, executes and supervises the nursing team in the implementation of dressings, acting in the prevention, evaluation and indication of the appropriate treatment for the injury.

Key words: Wounds and injuries, Healing, Health centers.

\section{RESUMEN}

Objetivo: Verificar la importancia del cuidado de enfermería en el cuidado de heridas en la atención primaria de salud. Métodos: Esta es una revisión de literatura integradora, realizada en las bases de datos de la Biblioteca Pública de la Biblioteca Nacional de Medicina de EE. UU. (PubMed) y la Biblioteca Virtual de Salud (BVS) a través de la asociación de descriptores, con estudios primarios como criterios de inclusión, disponible en su totalidad, publicado en los últimos 5 años, en cualquier idioma que cumpla con el tema. Resultados: Se realizó un análisis de 11 artículos que discutieron la importancia de la atención de enfermería en la atención de heridas en la atención primaria de salud, en el que se observó que la atención se realiza empíricamente y sin el uso de protocolos, identificó la escasez adecuado para realizar el tratamiento de heridas, así como el bajo conocimiento de los profesionales sobre el tratamiento, la conducta, la elección del vendaje y la asistencia brindada a los pacientes, asociada con la falta de capacitación de los profesionales y las deficiencias en su proceso de capacitación. Consideraciones finales: Los profesionales de enfermería juegan un papel extremadamente importante en el tratamiento de heridas, guían, ejecutan y supervisan al equipo de enfermería en la implementación de apósitos, actuando en la prevención, evaluación e indicación del tratamiento adecuado para la lesión.

Palabras clave: Heridas y lesiones, Curación, Centros de salud.

\section{INTRODUÇÃO}

Sendo a pele um dos maiores órgão do corpo humano, esta é responsável por realizar importantes funções ao corpo contra diversos agentes patológicos, algumas de suas funções consiste em regular a temperatura do corpo, funções sensorial, metabólica e excretora. Assim como os demais órgãos a pele está sujeita a sofrer alguns tipos de agressões como por exemplo as feridas que prejudicar o funcionamento do órgão (CARNEIRO CM, et al., 2010).

No Brasil, as feridas acometem a população de forma geral, independente de sexo, idade ou etnia, constituindo assim, um sério problema de saúde pública. O surgimento de feridas onera os gastos públicos e prejudica a qualidade de vida da população, por causar alterações que provocam a desmotivação e a incapacidade para o autocuidado, e para as atividades de vida e de convívio social (BEDINA LF, et al., 2014).

As feridas são identificadas de acordo a sua classificação, agente causal, profundidade, forma, tamanho, quantidade de exsudato, localização, aparência e o ambiente do tratamento (FONTES MMA e GAMA FN, 2011). O acompanhamento do processo de cicatrização da ferida é realizado após a avaliação da ferida por um enfermeiro periodicamente, por meio da avaliação da ferida é possível determinar o tipo de ferida, o tratamento adequado e as coberturas necessárias para cada momento do processo de cicatrização, o tratamento deve visar a prevenção de infecções e a cicatrização da ferida (ALCOFORADO CLGC e SANTO FHE, 2012).

De acordo com a lei do exercício profissional no 7498/86 é privativo do enfermeiro a organização e direção dos serviços e unidades de enfermagem, a assistência direta ao paciente que necessita de cuidados, a execução de maior complexidade técnica e que exijam conhecimento de base cientifica e capacidade de tomar decisões (BRASIL, 2012). Desse modo, o enfermeiro deve estar preparado para orientar e capacitar os técnicos de enfermagem para prestar uma assistência aos clientes que necessitam 
de cuidados em relação às feridas apresentadas, livre de risco e na satisfação pessoal daqueles que trabalham nesta prática (BUSANELLO J, et al., 2013).

O tratamento de feridas vem evoluindo com técnicas e medicamentos adequados para que se possam obter melhores resultados nas Unidades Básica de Saúde(UBS), visto que estas são as portas de entrada do sistema de saúde e consideradas uma prioridade na gestão do sistema, é importante que elas funcionem adequadamente, para que dessa forma a comunidade consiga resolver, com qualidade, a maioria dos seus problemas de saúde (BRASIL, 2012).

No cuidado às pessoas com feridas, é relevante elaborar Protocolo de Assistência aos Portadores de Feridas, em prol de minimizar os problemas decorrentes de uma técnica inadequada. Os protocolos visam instrumentalizar as ações dos profissionais e sistematizar a assistência a ser prestada ao portador de ferida, além de fornecer subsídios para a implementação deste tratamento (BRUM MLB, et al., 2015).

Segundo Jez RL e Brey C (2016), quando a equipe de enfermagem da UBS presta a assistência ao paciente com ferida de forma adequada e continua o tratamento se torna eficaz e a reabilitação mais rápida, para isso o profissional deve ter conhecimento quanto as formas de prevenção das feridas, a sua classificação, medicamentos utilizados, coberturas e o melhor tratamento para cada tipo de ferida. Diante disso, pretende-se com este estudo elencar na literatura evidências cientifica acerca da importância da assistência de enfermagem no cuidado deferidas na atenção primaria em saúde.

\section{MÉTODOS}

O estudo refere-se a uma revisão integrativa da literatura. Para a elaboração do estudo utilizou-se algumas etapas, tais como a definição do objetivo; estabelecimento de critérios de inclusão e exclusão; análise dos estudos selecionados e discussão dos resultados. Para nortear este estudo, formulou-se a seguinte questão: Qual a importância da assistência de enfermagem adequada a portadores de feridas na atenção primária de saúde?

A buscar dos estudos foi realizada nas bases de dados PubMed e BVS por meio da combinação dos seguintes descritores: Ferimentos e Lesões, Cicatrização, Centros de Saúde, nos idiomas português, inglês e espanhol nas mesmas bases.

Foram incluídos na busca os estudos primários, disponíveis em sua totalidade, publicados nos últimos cinco anos, em qualquer idioma que atendessem a temática do estudo. E foram excluídos da busca inicial capítulos de livros, resumos, textos incompletos, teses de doutorado, dissertações de mestrados e relatos técnicos. Os termos utilizados durante a pesquisa foram classificados e combinados nos bancos de dados BVS e PubMed, resultando nas seguintes estratégias específicas de cada base (Quadro 1).

Quadro 1 - Estratégias de busca nas bases de dados BVS e PubMed.

\begin{tabular}{|l|l|}
\hline Base & Link \\
\hline BVS & $\begin{array}{l}\text { +id:("bde-27487" OR "biblio-840478" OR "bde-25100" OR "bde-31414" OR } \\
\text { "bde-31975" OR "bde-31445" OR "bde-27177" OR "bde-27830" OR "bde- } \\
\text { 26908" OR "sus-31459" OR "bde-27600") }\end{array}$ \\
\hline PubMed & $\begin{array}{l}\text { (((("Wounds and Injuries"[Mesh])) OR "Wounds, Penetrating"[Mesh])) AND } \\
\text { (("Wound Healing"[Mesh]) OR "Wound Closure Techniques"[Mesh])) AND } \\
\text { (("Primary Health Care"[Mesh]) OR "Community Health Centers"[Mesh]) }\end{array}$ \\
\hline
\end{tabular}

Fonte: Carvalho BL, et al., 2019.

Os estudos incluídos na pesquisa foram pré-selecionados segundo os critérios de inclusão e exclusão nas bases de dado BVS e PubMed nas quais obteve-se um total de 11 artigos selecionados. Na busca geral na BVS obteve-se quinhentos e doze (512) estudos, ao realizar a filtragem dos estudos limitando-os em artigos com texto completo, nos idiomas Português, Espanhol e Inglês, realizados nos últimos cinco, obtevese cinquenta e um (51) estudos, ao analisar títulos e resumos apenas nove (9), foram condizentes com a questão desta pesquisa. 
Na Pubmed, como busca total foram encontrados duzentos e nove (209) estudos, ao realizar a filtragem em artigos com texto completo, nos idiomas Português, Espanhol e Inglês, realizados nos últimos cinco obtivemos quinze (15) estudos, destes foram analisados títulos e resumos e teve como resultado final dois (2) estudo.Na segunda fase os estudos foram analisados quanto ao potencial de participação no estudo, avaliando o atendimento à questão de pesquisa, bem como o tipo de investigação, objetivos, amostra, método, desfechos, resultados e conclusão, resultando em dez (11) artigos utilizados (Figura 1).

Figura 1 - Fluxo do processo de seleção dos estudos para a revisão integrativa.

Questão norteadora: Qual a importância da assistência de enfermagem adequada a portadores de feridas na atenção primária de saúde?

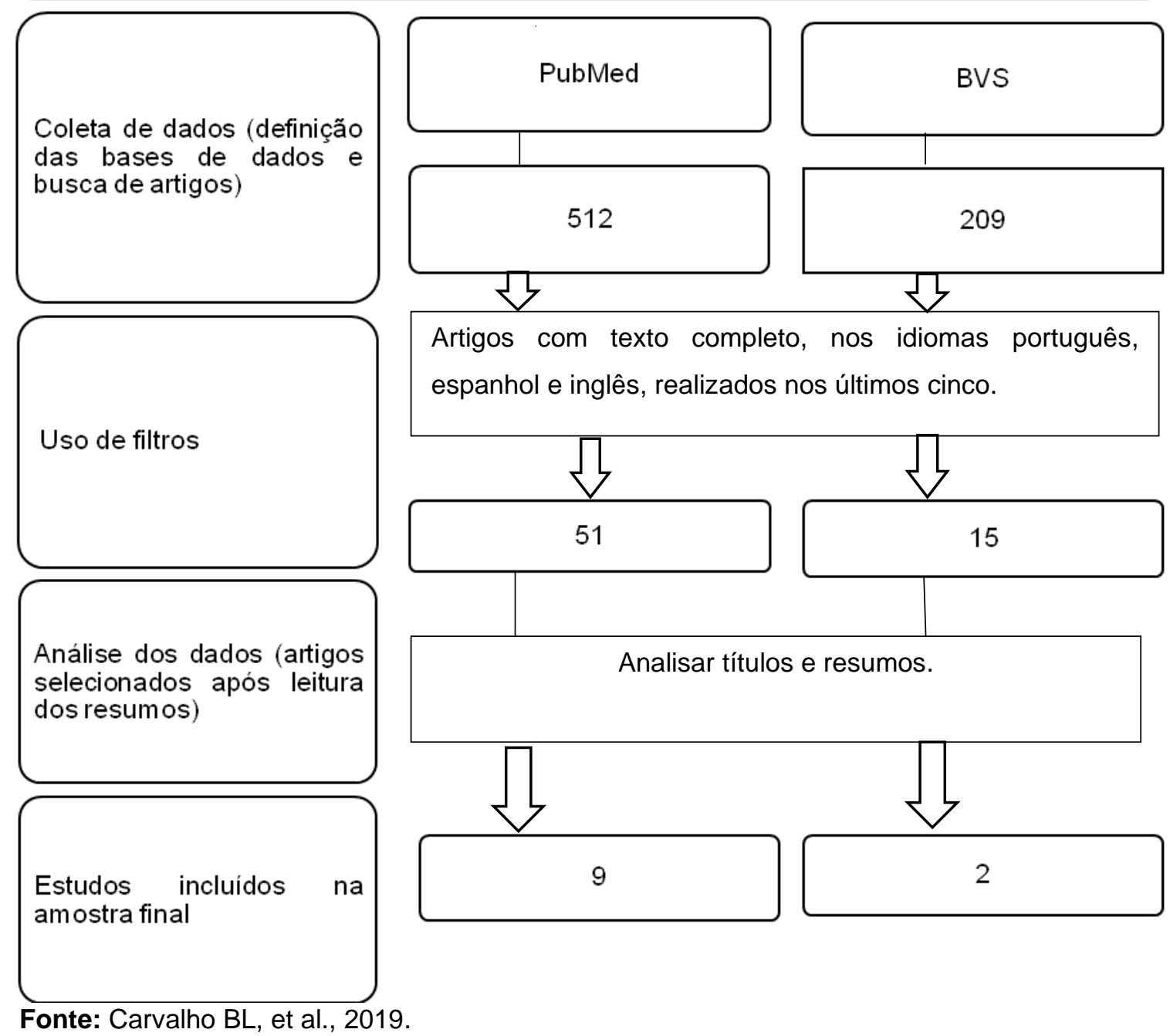

\section{RESULTADOS E DISCUSSÃO}

A principal linha de pesquisa investigada neste estudo versou sobre a importância da assistência de enfermagem adequada a portadores de feridas na atenção primária de saúde. Os 11 estudos incluídos nesta revisão foram organizados segundo os autores, delineamento da pesquisa, objetivo principal, amostra, principais resultados, nível de evidência e grau de recomendação para melhor compreensão e exposição dos estudos (Quadro 2). 
Quadro 2 - Organização dos estudos segundo os autores, delineamento da pesquisa, objetivo principal, amostra, principais resultados, nível de evidência e grau de recomendação.

\begin{tabular}{|c|c|c|c|c|c|}
\hline Autor & Objetivo principal & Amostral & Principais resultados & NE & GR \\
\hline Azevedo IC, et al., 2014 & $\begin{array}{l}\text { Identificar as dificuldades enfrentadas } \\
\text { pelos enfermeiros no cuidado às } \\
\text { pessoas com feridas oncológicas no } \\
\text { contexto da estratégia saúde da } \\
\text { Família. }\end{array}$ & 14 enfermeiros & $\begin{array}{l}\text { Identificou-se que os profissionais enfrentam } \\
\text { dificuldades na escolha do curativo, dos } \\
\text { medicamentos e limitações na operacionalização da } \\
\text { assistência, em função de problemas na organização } \\
\text { do serviço. }\end{array}$ & 5 & $A$ \\
\hline Agra G, et al., 2017 & $\begin{array}{l}\text { Verificar o conhecimento e prática de } \\
\text { enfermeiros no cuidado a pacientes } \\
\text { com feridas tumorais malignas. }\end{array}$ & 22 enfermeiros & $\begin{array}{l}\text { Os resultados permitiram identificar que os } \\
\text { enfermeiros apresentam lacunas no conhecimento de } \\
\text { conteúdos e técnicas sobre avaliação e tratamento de } \\
\text { pacientes com feridas. }\end{array}$ & 3 & $A$ \\
\hline Chibante CLP, et al., 2017 & $\begin{array}{l}\text { Descrever os saberes e práticas de } \\
\text { clientes no cuidado com feridas. }\end{array}$ & 20 clientes & $\begin{array}{l}\text { Destacou-se a dependência do cuidado por um } \\
\text { profissional de saúde e o uso de práticas alopáticas e } \\
\text { populares advindas de informações com familiares e } \\
\text { pessoas de convivência }\end{array}$ & 2 & $A$ \\
\hline Santos ICRV, et al., 2014 & $\begin{array}{l}\text { Caracterizar o atendimento de } \\
\text { portadores de feridas na Atenção } \\
\text { Primária. }\end{array}$ & $\begin{array}{l}93 \text { unidades de } \\
\text { saúde familiar }\end{array}$ & $\begin{array}{l}\text { A prevalência dos portadores de feridas foi de } 1,9 \% \text {, } \\
\text { as úlceras vasculares corresponderam a } 74,1 \% \text { das } \\
\text { feridas tratadas. }\end{array}$ & 2 & B \\
\hline Costa RKS, et al., 2016 & $\begin{array}{l}\text { Identificar o conhecimento dos } \\
\text { graduandos de enfermagem no } \\
\text { cuidado à pessoa com lesão cutânea. }\end{array}$ & 56 acadêmicos & $\begin{array}{l}\text { A dificuldade em associar teoria e prática foi uma das } \\
\text { principais limitações dos graduandos (46\%). }\end{array}$ & 3 & $B$ \\
\hline Vieira CPB, et al., 2017 & $\begin{array}{l}\text { Analisar evidências científicas sobre as } \\
\text { tecnologias utilizadas por enfermeiros } \\
\text { no tratamento de feridas }\end{array}$ & Bases de dados & $\begin{array}{l}\text { Foram analisados } 13 \text { artigos, dos quais } 8(61,5 \%) \\
\text { utilizaram tecnologia dura, } 4(30,8 \%) \text { leveduras e } 1 \\
(7,7 \%) \text { leve, sendo que entre as duras o uso de } \\
\text { coberturas foi o que mais se destacou, seguido de } \\
\text { sistema de medição digital e biomarcadores. }\end{array}$ & 1 & $A$ \\
\hline
\end{tabular}

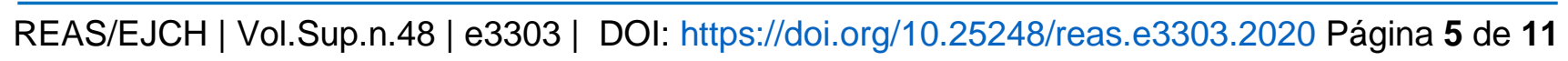




\section{Revista Eletrônica Acervo Saúde / Electronic Journal Collection Health | ISSN 2178-2091}

\begin{tabular}{|c|c|c|c|c|c|}
\hline Autor & Objetivo principal & Amostral & Principais resultados & NE & GR \\
\hline Busanello J, et al., 2013 & $\begin{array}{l}\text { Conhecer as tecnologias de cuidado } \\
\text { implementadas no cuidado a indivíduos } \\
\text { portadores de feridas em unidades de } \\
\text { atenção primária. }\end{array}$ & 8 enfermeiros & $\begin{array}{l}\text { A análise temática foram estruturadas três categorias: } \\
\text { comunicação e vínculo na mediação do cuidado aos } \\
\text { indivíduos portadores de feridas; sistematização da } \\
\text { assistência de enfermagem aos indivíduos portadores } \\
\text { de feridas; e terapias tópicas no cuidado aos } \\
\text { indivíduos portadores de feridas. }\end{array}$ & 5 & $A$ \\
\hline Brum MIB, et al., 2015 & $\begin{array}{l}\text { Conhecer se os enfermeiros utilizam os } \\
\text { protocolos no cuidado aos usuários } \\
\text { com feridas crônicas. }\end{array}$ & 16 enfermeiros & $\begin{array}{l}\text { Poucos enfermeiros aplicam os protocolos existentes } \\
\text { e, ocasionalmente exercitam sua autonomia. }\end{array}$ & 2 & A \\
\hline Ferreira AM, et al., 2013 & $\begin{array}{l}\text { Identificar o conhecimento, a prática e } \\
\text { fontes de informações de graduandos } \\
\text { de enfermagem sobre o cuidado a } \\
\text { portadores de feridas. }\end{array}$ & $\begin{array}{l}68 \text { graduandos } \\
\text { de enfermagem }\end{array}$ & $\begin{array}{l}\text { Constatou-se que a maioria dos acadêmicos não } \\
\text { utiliza fontes de informações para atualização sobre o } \\
\text { assunto. Os resultados demonstraram que o nível de } \\
\text { conhecimento sobre o cuidado a portadores de feridas } \\
\text { é baixo. }\end{array}$ & 5 & $B$ \\
\hline Oliveira BGRB, et al., 2013 & $\begin{array}{l}\text { Realizar um estudo epidemiológico dos } \\
\text { pacientes com feridas crônicas. }\end{array}$ & $\begin{array}{l}\text { Exame clínico } \\
\text { de } 186 \\
\text { pacientes }\end{array}$ & $\begin{array}{l}\text { O maior percentual de pacientes está na faixa etária } \\
\text { entre } 57-69 \text { anos }(28 \%) \text {, é do sexo feminino (54\%) e } \\
\text { apresenta comorbidades como hipertensão arterial e } \\
\text { diabetes ( } 73 \%) \text {. A maior parte tem apenas uma ferida } \\
(65 \%) \text { de etiologia venosa ( } 51 \%) \text {. }\end{array}$ & 4 & B \\
\hline Silva MH, et al., 2012 & $\begin{array}{l}\text { Discutir o manejo clínico de úlceras } \\
\text { venosas realizado na atenção primária } \\
\text { à saúde. }\end{array}$ & 25 usuários & $\begin{array}{l}\text { São usados para a limpeza produtos que agridem o } \\
\text { tecido de granulação, como coberturas com várias } \\
\text { substâncias, dentre elas o óleo de girassol e pomadas } \\
\text { antibióticas; a maioria dos usuários não utiliza } \\
\text { medidas para controle do edema. }\end{array}$ & 5 & A \\
\hline
\end{tabular}

Fonte: Carvalho BL, et al., 2019. 
A Tabela 1 mostra que a fonte online BVS com $81 \%$ da amostra, foi a que mais disponibilizou estudos sobre a temática proposta em relação a outra fonte online PubMed (18\%) foi a que menos disponibilizou estudos sobre o tema estudado.

Tabela 1 - Distribuição dos estudos incluídos na amostra referentes à fonte online de publicação.

\begin{tabular}{ccc}
\hline Fonte online & Número absoluto & $\%$ \\
\hline PubMed & 2 & $18 \%$ \\
\hline BVS & 9 & $81 \%$
\end{tabular}

Fonte: Carvalho BL, et al., 2019..

Quanto aos periódicos, no Quadro 3 observa-se que houve a distribuição de forma uniforme dos estudos. Um total de 11 revistas tiveram apenas um artigo utilizado cada uma que somando contribuíram imensamente para o trabalho.

Quadro 3 - Distribuição dos estudos incluídos na amostra, referentes ao nome do periódico.

\begin{tabular}{|l|l|}
\hline \multicolumn{1}{|c|}{ Autor } & \multicolumn{1}{c|}{ Nome do periódico } \\
\hline Azevedo IC, et al., 2014 & Revista Brasileira de Cancerologia \\
\hline Agra G et al., 2017 & J. res.: fundamental \\
\hline Chibante CLP et al., 2017 & Revista Cuidarte \\
\hline Santos ICRV et al., 2014 & Escola Anna Nery \\
\hline Costa RKS et al., 2016 & Rev Rene \\
\hline Vieira CPB et al., 2017 & Rev Enferm UFPI \\
\hline Busanello J et al., 2013 & Rev Enferm UFSM \\
\hline Brum MIB et al., 2015 & Rev Enferm UFSM \\
\hline Ferreira AM et al., 2013 & Esc Anna Nery \\
\hline Oliveira BGRB et al., 2013 & Rev. enferm. UERJ \\
\hline Silva MH et al., 2012 & Acta Paul Enferm \\
\hline
\end{tabular}

Fonte: Carvalho BL, et al., 2019.

Ao analisar os estudos, de acordo com a temática abordada por cada um, pode-se observar que estes em sua maioria têm em comum o enfoque principal dado a importância da assistência de enfermagem adequada na atenção primaria em saúde ao paciente com ferida. Com base nos achados, emergiram as seguintes categorias:

\section{Ferramenta de cuidar baseado em evidências}

Os protocolos clínicos têm o objetivo de sistematizar a assistência de enfermagem no cuidado de feridas, bem como contribuir para a redução dos custos em saúde. Respaldando as condutas de avaliação, diagnóstico, planejamento, cuidado, tratamento, evolução e registro de todos os dados do usuário, sendo está uma ferramenta que oferece maior autonomia aos profissionais, resultado eficaz para o paciente e custos menores para a instituição (BRUM MLB, et al., 2015).

Ha evidencias que relatam que os protocolos quando utilizados no cuidado as feridas proporcionam melhoria da qualidade do atendimento de enfermagem, resultando em benefícios para o paciente e criando oportunidades para futuras intervenções visando à melhoria contínua do serviço que será garantida após 
sensibilização da equipe para sua utilização pois os protocolos promovem orientações para a equipe de enfermagem, estado de saúde do cliente e características que proporcionará ao mesmo um tratamento eficaz e uma reabilitação completa (OLIVEIRA FBM e PAULA ABR, 2015).

A utilização dos protocolos possibilita a identificação da ferida, a sua classificar e o tratamento adequado. Segundo Costa AP, et al. (2019) as feridas podem ser classificação de acordo com a sua etiológica, podendo ser cirúrgicas, traumática ou ulcerativas, de acordo com o tempo, podendo ser feridas crônica ou aguda, ou de acordo com o grau de contaminação que pode ser uma feridas limpas, feridas potencialmente contaminadas/limpa-contaminadas, feridas contaminadas ou uma feridas infectadas.

A mensuração da ferida é realizada por meio da medida bidimensional na qual mede-se o comprimento e a largura das feridas por meio de traçados lineares ou por meio da medida tridimensional que abrange além do comprimento e da largura, a profundidade da ferida. Segundo o autor o processo de cicatrização de uma ferida pode ocorrer de cinco formas, sendo estas a cicatrização por primeira intenção, cicatrização por segunda intenção, cicatrização por terceira intenção, cicatrização no diabetes mellitus e a cicatrização em coagulopatias (COSTA AP, et al., 2019).

\section{Características das lesões das ABS e tratamentos}

De acordo com Vieira CPB, et al. (2017) cerca de 55,8\% dos pacientes atendidos na atenção básica da cidade de Teresina (PI) eram idosos com idade entre 60 a 70 anos, destes $91,7 \%$ tinham uma ou mais doenças de base. Resultado similar foi identificado no estudo realizado por Ribeiro DFS (2019) que identificou uma maior prevalência de idosos (66,7\%) com idade média entre 60 e 71 anos, no estudo a maioria dos idosos foram diagnósticos com doenças crônicas prevalentes, tais como hipertensão arterial sistêmica, diabetes mellitus ou doenças vasculares periféricas.

Corrobora com os achados citados o estudo realizado por Campoi ALM, et al. (2019) que também identificou maior prevalência de feridas em idosos atendidos na atenção primaria de saúde, a doença de base prevalente foi a hipertensão arterial $(70,1 \%)$, e cerca de $35 \%$ dos idosos apresentavam mais de uma lesão que em sua maioria eram crônicas e/ou lesão por pressão.

De acordo com os estudos as regiões mais acometidas pelas feridas é a região sacral (todas do tipo lesão por pressão), região plantar (80\% úlceras diabéticas) e o terço distal da perna. Segundo Feitosa MNL, et al. (2017) o pé diabético representa uma das complicações mais graves e dispendiosas da doença, sendo responsável por $70 \%$ de todas as amputações realizadas por causa não traumática, sendo que $85 \%$ dessas são precedidas por uma úlcera no pé.

Segundo Oliveira BGRB, et al. (2013), o processo de cicatrização das feridas depende do tratamento adequado, desta forma a escolha correta dos medicamentos e coberturas deve visar tanto a cicatrização quanto a prevenção de infecções, para isso ressalta-se a importância do conhecimento dos profissionais as diversas coberturas disponíveis, utilizando-as como complementação do tratamento mais adequado. É importante conhecer os benefícios da cobertura a ser aplicada, as contraindicações, indicações e o custo, de forma a selecionar a mais adequada para cada etapa do processo terapêutico.

Algumas características devem ser consideradas para a escolha da cobertura mais apropriada para manter 0 ambiente propicio para a reparação tissular. Tais como manter umidade na interface ferida/cobertura, remover o excesso de exsudato, permitir a troca gasosa, promover isolamento térmico, proporcionar proteção contra infecção, ser isento de partículas e contaminações e permitir a remoção sem causar traumas (SANTOS ICRV, et al., 2014).

Ao analisar os estudos pode-se observar que os produtos mais utilizados no tratamento das feridas na tenção básica de saúde é o Hidrogel® e os ácidos graxos essenciais, o qual é disponibilizado em pouca quantidade para atenção primaria, as coberturas não são fornecidas para a atenção primaria e a falta do produto ou a compra inadequada leva à interrupção do tratamento, nesse sentido, reforça-se a importância do enfermeiro no gerenciamento e na assistência direta ao paciente com lesão (SANTOS ICRV, et al., 2014). Resultado similar foi evidenciado no estudo de Resende NM, et al. (2017) que em entrevistas realizadas com a equipe responsável diretamente pelos cuidados com feridas crônicas, foi implicada como principal dificuldade no cuidado a falta de material adequado.

REAS/EJCH | Vol.Sup.n.48 | e3303 | DOI: https://doi.org/10.25248/reas.e3303.2020 Página 8 de 11 
O estudo identificou que houve uma discrepância do autocuidado entre os pacientes e sua relação direta no processo de cura, visto que os pacientes com melhor renda usam, por conta própria, materiais mais eficazes no tratamento de feridas, uma vez que o município não os oferece regularmente.

Azevedo IC, et al. (2014), ressalta em seu estudo alguns dos produtos mais utilizados no tratamento de feridas na atenção primaria, no estudo foram citados: solução fisiológica a 0,9\%, gaze, óleo de girassol, colagenase $^{\circledR}$, atadura, iodopovidona ${ }^{\circledR}$, papaína, fita hipoalergênica e fibrinolisina ${ }^{\circledR}$.

De acordo com os entrevistados, os produtos para limpeza e cobertura das feridas malignas disponíveis nas unidades de saúde da família eram: solução fisiológica 0,9\%; gaze; esparadrapo; atadura, sulfato de neomicina; iodopovidona. e outros materiais e medicamentos como fita hipoalergênica; óleo de girassol; papaína; colagenase ${ }^{\circledR}$; e fibrinolisina eram custeados pelos próprios pacientes quando indicados para 0 tratamento das lesões.

No estudo realizado por Khatcherian GP, et al. (2018) houve a predominância da utilização da solução fisiológica $(\mathrm{NaCl} 0,9 \%)$ morna $\left(37^{\circ} \mathrm{C}\right)$ aplicada em jato para realizar a limpeza da ferida, evitando a fricção da gaze diretamente sobre a lesão. O Metronidazol na forma de creme foi aplicado na pele e tecido vivo, destruindo os compostos vegetativos como as bactérias e impedindo seu crescimento. Depois o membro inferior foi ocluído por gazes e enfaixado, as trocas foram feitas semanalmente, segundo o autor 0 tratamento mostrou-se eficiente e houve a cicatrização da ferida.

Campoi ALM, et al. (2019) ao relatar o tratamento de ferida por uma equipe de enfermagem ressalta que houve a utilização de alginato de cálcio e sódio, hidrogel com alginato e rayon e para umidificar a ferida utilizou óleo amaciante com AGE.

De acordo com o estudo foram realizadas 12 aplicações de Laser de Baixa Potência (LBP), com 2 dias de intervalo entre cada aplicação, durante cerca de 60 minutos no início do tratamento devido a extensão da lesão, segundo o autor as sessões de LBP acelerou o processo de cicatrização e reduziu o desconforto ocasionado pela ferida.

Ribeiro DFS (2019) ao buscar identificar o perfil de cuidados implementados aos usuários portadores de lesões crônicas de uma Unidade Básica de Saúde no município do Rio de Janeiro/RJ identificou que a antissepsia das lesões foram realizadas em $73,3 \%$ das vezes através da combinação do uso de cloreto de sódio (soro fisiológico) a 0,9\% e solução aquosa de polihexanida (PHMB), segundo o autor como 63,3\% das lesões eram colonizadas ou infectadas, entende-se que o uso de PHMB foi efetivo na maior parte das vezes, tendo em vista seu potencial antimicrobiano ao biofilme da lesão.

No estudo pode-se identificar ainda que em $20 \%$ das lesões eram utilizadas combinações de mais de uma cobertura especial, houve predomínio do uso de três coberturas: hidrogel com alginato de cálcio e sódio, gaze rayon embebida com óleos e ácidos graxos essenciais (AGE) e alginato de cálcio em placa, utilizadas, respectivamente em 33,3\%, 25\% e em 16,7\% das lesões (SANTOS TL, et al., 2019).

Em seguida encontraram-se a malha não aderente com petrolatum (8,3\%), a hidrofibra de carboximetilcelulose sódica e prata $(5,6 \%)$, o carvão ativado com prata em placa $(5,5 \%)$, a espuma de poliuretano impregnado com prata $(2,8 \%)$ e a bota de Unna $(2,8 \%)$, havendo desta forma coerência com os tipos de tecidos e demais características frequentes nos leitos das lesões (RIBEIRO DFS, 2019).

Segundo Santos ICRV, et al. (2014) a utilização dos produtos adequados para o tratamento das feridas está diretamente relacionada ao custo-efetivo, uma vez que implica menos troca de curativo e, consequentemente, menos dor, menos risco de contaminação e de infecção pelas técnicas de curativo executadas, ou de exposição ao meio ambiente. Isso diminui o ônus relacionado ao tratamento, no entanto o que se pode-se observar é que essa não é a realidade das Unidades Primaria de Saúde.

\section{Assistência de enfermagem no tratamento de lesões}

O profissional de enfermagem tem importante papel no tratamento e prevenção de lesões, o enfermeiro deve ser responsável pela supervisão, incluindo a prescrição de produtos adequados, a orientação, o acompanhamento, o desenvolvimento de processos educativos com os profissionais, culminando com a avaliação dos resultados (VIEIRA CPB, et al., 2017; BUSANELLO J, et al., 2013). 
No entanto ao avaliar o conhecimento de enfermeiros da atenção primária em saúde sobre avaliação e tratamento de feridas, observou-se que a maioria dos entrevistados apresentam lacunas no conhecimento de conteúdos e técnicas sobre avaliação e tratamento de pacientes com feridas (AGRA G, et al., 2017). Observou-se ainda que os profissionais enfrentam dificuldades na escolha do curativo, dos medicamentos a serem utilizados nas lesões e limitações na operacionalização da assistência, em função de problemas na organização do serviço (AZEVEDO IC, et al., 2014).

Estudo realizado por Resende NM, et al. (2017) observou que todos os profissionais que participaram da pesquisa tinham dificuldade em relação as caracterizar dos estágios de cicatrização das feridas. Segundo 0 autor os profissionais devem ser capacitados para avaliar características como o tamanho da ferida, o local de ocorrência, margens, presença de exsudato, edema, hiperpigmentação, dor, presença de dermatite associada, pulsos, doença avançada, esfacelo e evolução da ferida.

Uma pesquisa realizada por Silva DRVP, et al. (2020) identificou a ausência ou o desconhecimento de protocolos para o manuseio de feridas em uma UBS, de acordo com o estudo com a ausências dos protocolos prejudicar a avaliação, a classificação da ferida e o uso de técnicas assépticas, práticas que, quando feitas corretamente e de maneira completa, orientam a funcionalidade do tratamento e auxiliam sua readaptação.

De acordo com Caveião $C$, et al. (2018) os enfermeiros da atenção primária não têm conhecimento suficiente para indicação das coberturas, no estudo pode-se identificar que os profissionais não detêm o devido conhecimento para utilização e prescrição da cobertura.

No estudo a indicação da cobertura, $79,2 \%$ referiu que se destina a controlar o exsudato e o tecido não viável, e a indicação com maior porcentagem de acertos para utilização em feridas foi a cobertura hidrofibra de prata e hidrocoloide com prata. No entanto a maioria dos profissionais não cita a função de desbridamento autolítico nas coberturas que têm essa função, como hidrocoloide, hidrocoloide com prata, alginato de cálcio, espuma com prata, hidrofibra e o hidrogel.

A deficiência no conhecimento do tratamento e avaliação das lesões é semelhante em graduandos de enfermagem, estes mostraram baixo nível de conhecimento, dificuldade em associar teoria e prática e limitações quanto aos aspectos do cuidado as feridas (FERREIRA AM, et al., 2013). Esta deficiência no conhecimento é observada desde a graduação dos acadêmicos, com baixa carga horária da disciplina dedicada ao ensino do cuidado de pacientes com feridas, não há estimulo aos acadêmicos a buscarem fontes de atualização e os alunos não participam de treinamentos ou estágios extracurriculares (COSTA RKS, et al., 2016).

Diante dos resultados, fica evidente a necessidade de investimentos no ensino de graduação do referido curso, bem como o treinamento da equipe de enfermagem, educação permanente e a implantação dos protocolos assistenciais que norteiem a prática de métodos avaliativos e terapêuticos para o cuidado com pessoas com lesões baseado em evidências científicas (SILVA MH, et al., 2012; CHIBANTE CLP, et al., 2017). Segundo Silva DRVP, et al. (2020) o acesso dos profissionais a recursos materiais adequados, a treinamentos específicos e ao desenvolvimento de um trabalho interdisciplinar são fatores indispensáveis para que possam ser viabilizadas as condições necessárias para o estabelecimento de condutas terapêuticas eficazes no tratamento das feridas.

O autor ressalta ainda há necessidade da realização de educação continuada, para incorporar sempre novos conhecimentos, bem como estruturação das unidades de atenção à saúde, com recursos humanos e materiais necessários, criação e implantação de protocolos assistenciais que orientem a prática de condutas terapêuticas eficazes no cuidado a pessoas com feridas, familiares e cuidadores, para que seja clarificada a responsabilidade e a atuação de cada profissional que lida frente ao cuidado de feridas (MORAES AMM, et al., 2018).

\section{CONSIDERAÇÕES FINAIS}

Por meio do estudo pode-se identificar a importância dos profissionais de enfermagem no tratamento de feridas na atenção primária em saúde pois estes desempenham um papel de extrema importância que orienta, executa e supervisiona a equipe de enfermagem na realização de curativos, atuando na prevenção,

REAS/EJCH | Vol.Sup.n.48 | e3303 | DOI: https://doi.org/10.25248/reas.e3303.2020 Página 10 de 11 
avaliação e indicação do tratamento adequado para a lesão. Desta forma ressalta-se a importância que os profissionais da enfermagem tenham conhecimento para acompanhar a evolução da lesão de forma eficaz, com a escolha do procedimento e dos instrumentos adequado. Destaca-se como limitação do estudo a existência de poucos artigos específicos sobre o tratamento de feridas na atenção primária de saúde, que impossibilitou maiores comparações e aprofundamentos na temática.

\section{REFERÊNCIAS}

1. AGRA G, et al. Conhecimento e prática de enfermeiros no cuidado a pacientes com feridas tumorais malignas. Rev Cuid., 2017; 8(3): 1849-62.

2. ALCOFORADO CLGC e SANTO FHE. Saberes e práticas dos clientes com feridas: um estudo de caso no município de cruzeiro do Sul, Acre. Rev. Min. Enferm., 2012; 16(1): 11-17.

3. AZEVEDO IC, et al. Conhecimento de Enfermeiros da Estratégia Saúde da Família sobre Avaliação e Tratamento de Feridas Oncológicas. Rev. Brasileira de Cancerologia. 2014; 60(2): 119-127.

4. BRASIL. Ministério da Saúde. Secretaria de Atenção à Saúde Departamento de Atenção Básica. Política Nacional de Atenção Básica. Brasília, DF, 2012 (Série E. Legislação em Saúde).

5. BUSANELLO J, et al. Assistência de enfermagem a portadores de feridas: tecnologias de cuidado desenvolvidas na atenção primária. Rev Enferm UFSM. 2013; 3(1):175-184.

6. BRUM MLB, et al. Protocolo de assistência de enfermagem a pessoas com feridas como instrumento para autonomia profissional. Rev Enferm UFSM. 2015; 5(1): 50-57.

7. BEDINA LF, et al. Estratégias de promoção da autoestima, autonomia e autocuidado das pessoas com feridas crônicas. Rev Gaúcha Enferm., 2014; 35(3): 61-67.

8. CARNEIRO CM, et al. Tratamento de feridas: assistência de enfermagem nas unidades de atenção primária à saúde. Rev. Enf Integrada - Ipatinga, Unileste-MG. 2010; 3(2).

9. CAVEIÃO $\mathrm{C}$, et al. Conhecimento do enfermeiro da atenção primária à saúde sobre a indicação de coberturas especiais. ESTIMA, Braz. J. Enterostomal Ther., 2018; 16: e3118.

10. CARVALHO BL, et al. Assistência de enfermagem a pacientes com estoma intestinal. Revista Eletrônica Acervo Saúde (REAS). 2019; S24: e604.

11. CHIBANTE CLP, et al. Saberes e práticas no cuidado centrado na pessoa com feridas. Esc Anna Nery. 2017; 21(2):190-89.

12. COSTA RKS, et al. Graduandos de enfermagem: conhecimento sobre o cuidado à pessoa com lesão cutânea. Rev Enferm UFPI. 2016; 5(1): 10-16.

13. COSTA AP, et al. Protocolo de reconhecimento e tratamento de feridas na atenção primaria - Porto Velho: Centro Universitário São Lucas, 2019.

14. CAMPOI ALM, et al. Assistência de enfermagem a pacientes com feridas crônicas: um relato de experiência. REFACS (online) 2019; 7(2): 248-255.

15. FERREIRA AM, et al. Conhecimento e prática de acadêmicos de enfermagem sobre cuidados com portadores de feridas. Esc Anna Nery. 2013; 17(2): $211-219$.

16. FEITOSA MNL, et al. Assistência de enfermagem na atenção primária ao paciente com risco potencial de desenvolver pé diabético: Uma revisão bibliográfica. Rev. UNINGÁ, Maringá. 2017; 54(1): 185-196.

17. FONTES MMA e GAMA FN. Análise da técnica do curativo no tratamento de feridas em unidades de atenção primária à saúde no município de coronel fabriciano - MG. Rev. Enf Integrada - Ipatinga. 2011; 4(2).

18. JEZ RL e BREY C. Curativos Especiais: Capacitação para equipe de Enfermagem em uma Unidade básica de saúde. Anais do XI EVINCI - Centro Universitário Autônomo do Brasil - UniBrasil, 2016.

19. MORAES AMM, et al. A importância da atuação do enfermeiro como gestor na organização e administração da atenção básica: uma revisão integrativa. Revista Eletrônica Acervo Saúde (REAS). 2018; S10: S912-S917.

20. OLIVEIRA BGRB, et al. Panorama epidemiológico e clínico de pacientes com feridas crônicas tratados em ambulatório. Rev. enferm. 2013; 21(1): 612-7.

21. OLIVEIRA FBM, PAULA ABR. Protocolo clínico para úlceras por pressão: uma ferramenta assistencialista para a práxis de Enfermagem. ReOnFacema. 2015; 1(1): 17-24.

22. KHATCHERIAN GP, et al. Assistência de enfermagem integral e humanizada ao paciente portador de ferida em membros inferiores. Revista Enfermagem Atual. 2018; 85.

23. RESENDE NM, et al. Cuidado de pessoas com feridas crônicas na Atenção Primária à Saúde. J Manag Prim Heal Care. 2017; 8(1): 99-108.

24. RIBEIRO DFS. Gestão do cuidado a usuários com feridas crônicas na Atenção Básica. Revista Enfermagem Atual in Derme. 2019; 90-28.

25. SANTOS ICRV, et al. Caracterização do atendimento de pacientes com feridas na Atenção Primária. Rev Rene. 2014; 15(4): 613-20.

26. SANTOS TL, et al. Terapia por pressão negativa no tratamento de feridas. Revista Eletrônica Acervo Saúde (REAS). 2019; s31: e1231.

27. SILVA MH, et al. Manejo clínico de úlceras venosas na atenção primária à saúde. Acta Paul Enferm., 2012; 25(3): 329-33.

28. SILVA DRVP, et al. Intervencão de enfermagem na avaliação e tratamento de feridas em uma estratégia de saúde da família. Rev. Universidade Estadual do Piaui. 2020; 20(4).

29. VIEIRA CPB, et al. Tecnologias utilizadas por enfermeiros no tratamento de feridas. Rev Enferm UFPI. 2017; 6(1): 65-70. 